Research Paper

\title{
A Plasmonic Gold Nanostar Theranostic Probe for In Vivo Tumor Imaging and Photothermal Therapy
}

\author{
Yang Liu1,2,3†, Jeffrey R. Ashton'†, Everett J. Moding4, Hsiangkuo Yuan,2, Janna K. Register1,2, Andrew M. \\ Fales ${ }^{1,2}$, Jaeyeon Choi5, Melodi J. Whitley4, Xiaoguang Zhao5, Yi Qi', Yan Ma7, Ganesan Vaidyanathan ${ }^{5}$, \\ Michael R. Zalutsky ${ }^{5,6}$, David G. Kirsch ${ }^{4,7}$, Cristian T. Badea ${ }^{\natural}$, Tuan Vo-Dinh ${ }^{1,2,3 凶}$ \\ 1. Fitzpatrick Institute for Photonics, Duke University, Durham, NC, 27708, United States \\ 2. Department of Biomedical Engineering, Duke University, Durham, NC, 27708, United States \\ 3. Department of Chemistry, Duke University, Durham, NC, 27708, United States \\ 4. Department of Pharmacology and Cancer Biology, Duke University Medical Center, Durham, NC, 27710, United States \\ 5. Department of Radiology, Duke University Medical Center, Durham, NC, 27710, United States \\ 6. Center for In Vivo Microscopy, Department of Radiology, Duke University Medical Center, Durham, NC 27710, United States \\ 7. Department of Radiation Oncology, Duke University Medical Center, Durham, NC, 27710, United States \\ † These authors contributed equally to this work \\ $\bowtie$ Corresponding authors: tuan.vodinh@duke.edu, cristian.badea@duke.edu
}

(C) 2015 Ivyspring International Publisher. Reproduction is permitted for personal, noncommercial use, provided that the article is in whole, unmodified, and properly cited. See http://ivyspring.com/terms for terms and conditions.

Received: 2015.02.24; Accepted: 2015.04.12; Published: 2015.05.23

\begin{abstract}
Nanomedicine has attracted increasing attention in recent years, because it offers great promise to provide personalized diagnostics and therapy with improved treatment efficacy and specificity. In this study, we developed a gold nanostar (GNS) probe for multi-modality theranostics including surface-enhanced Raman scattering (SERS) detection, x-ray computed tomography (CT), two-photon luminescence (TPL) imaging, and photothermal therapy (PTT). We performed radiolabeling, as well as CT and optical imaging, to investigate the GNS probe's biodistribution and intratumoral uptake at both macroscopic and microscopic scales. We also characterized the performance of the GNS nanoprobe for in vitro photothermal heating and in vivo photothermal ablation of primary sarcomas in mice. The results showed that 30-nm GNS have higher tumor uptake, as well as deeper penetration into tumor interstitial space compared to $60-\mathrm{nm}$ GNS. In addition, we found that a higher injection dose of GNS can increase the percentage of tumor uptake. We also demonstrated the GNS probe's superior photothermal conversion efficiency with a highly concentrated heating effect due to a tip-enhanced plasmonic effect. In vivo photothermal therapy with a near-infrared (NIR) laser under the maximum permissible exposure (MPE) led to ablation of aggressive tumors containing GNS, but had no effect in the absence of GNS. This multifunctional GNS probe has the potential to be used for in vivo biosensing, preoperative CT imaging, intraoperative detection with optical methods (SERS and TPL), as well as image-guided photothermal therapy.
\end{abstract}

Key words: Gold nanostars, photothermal therapy, nanoparticles, tumor imaging, SERS, CT, biodistribution, theranostics

\section{Introduction}

Nanotheranostics, in which diagnostic and therapeutic functions are combined into a single nanometer-sized agent, provides an attractive means to improve cancer management by allowing tumor identification, real-time tracking of biodistribution, image-guided specific therapy, and continuous monitoring of therapeutic response.[1-6] Gold nanoparticles (e.g., nanorods, nanoshells, nanocages, and 
nanostars) have received great attention as nanotheranostic agents due to their unique properties including strong localized surface plasmon resonance, in vivo stability, well-established biocompatibility, and facile surface modification via thiol-gold chemical bonding.[4-7] Gold nanoparticles can be designed to have strong surface plasmon resonance in the near-infrared (NIR) region, which is considered to be the optimal optical window for biomedical imaging and therapy due to the low optical attenuation coefficients of water and other tissue chromophores in this spectral range (700-900 nm).[8] Gold nanoparticles can also be used for surface-enhanced Raman spectroscopy (SERS), multiphoton microscopy, photothermal therapy (PTT), photodynamic therapy (PDT), and other applications.[9-15] SERS combines surface plasmon resonance and "fingerprint" Raman spectroscopy to provide a sensing method with high sensitivity and specificity.[16-19] The SERS enhancement factor for gold nanoparticles is typically $10^{7}-10^{9}$ and can reach $10^{15}$ at "hot spots," enabling single-molecule detection.[20] PTT uses gold nanoparticles as a transducer to convert NIR light to heat and generate a localized temperature increase. The distinct advantage of PTT with gold nanoparticles is that the NIR light only causes heating within tissues that have gold nanoparticle accumulation. Tumors tend to have high uptake of nanoparticles due to the enhanced permeability and retention (EPR) effect,[21, 22] so they experience large temperature increases with NIR light exposure; surrounding tissues, which have minimal nanoparticle uptake, experience little to no change in temperature with NIR exposure. Thus, the gold nanoparticle-enhanced PTT method can specifically target tumors more effectively than traditional chemotherapy. Gold nanoparticles can also be used as contrast agents for x-ray computed tomography (CT) because gold exhibits high x-ray attenuation ( 2.7-times higher attenuation than iodine per mass).[23] Furthermore, gold nanoparticles can be functionalized with a wide variety of molecules suitable for magnetic resonance imaging (MRI), positron emission tomography (PET), single-photon emission computed tomography (SPECT), and optical imaging.[24] Therefore, gold nanoparticle theranostic probes could be used to perform pre-treatment scans with CT, MRI, or PET; in situ tumor detection using optical imaging and SERS; effective cancer PTT therapy using NIR light; targeted radiotherapy with linked radioisotopes; and therapeutic response monitoring with various imaging modalities.

Over the last two decades, our laboratory has devoted extensive effort to develop the SERS technique for chemical and biological sensing using various metallic nanostructures.[18, 25-28] Recently, our group has developed a unique surfactant-free method for the synthesis of gold nanostars (GNS) with tip-enhanced plasmonics.[29] This novel synthetic method produces GNS without the use of toxic surfactants traditionally required for GNS synthesis, which improves the biocompatibility of the resulting GNS. The GNS contain multiple sharp spikes, which act like lightning rods to dramatically enhance the local electromagnetic field. This design results in stronger SERS enhancement, larger two-photon action cross-section, and higher photothermal conversion efficiency than exhibited by other gold nanoparticle shapes.[29-31] Therefore, GNS have the potential to be used as a highly effective multimodality theranostic nanoprobe. In a previous in vitro study, we developed a quintuple-modality plasmonic nanoprobe based on GNS for SERS, MRI, CT, two-photon luminescence (TPL) and PTT.[32] We have also used GNS to investigate nanoparticle penetration through the blood-brain-barrier in brain tumors.[13] Although various properties of GNS have been investigated, their in vivo biodistribution, tumor uptake, intratumoral distribution, and feasibility for in vivo CT imaging, SERS detection, and tumor PTT ablation have not yet been fully investigated.

In this study, we demonstrated the in vivo application of the multifunctional GNS probe for both imaging and therapy. We performed in vivo GNS tracking using TPL, CT, and radiolabelling to examine how nanoparticle size and injection dose affect their biodistribution and intratumoral distribution. We also showed in vivo SERS detection of GNS accumulated within a primary sarcoma through the intact skin. Furthermore, we characterized the in vitro performance of GNS for PTT and demonstrated efficient in vivo tumor ablation. These results confirm that the developed multifunctional GNS can be applied for in vivo tracking at different resolution scales, as well as for image-guided PTT for specific cancer treatment.

\section{Materials and Methods}

\section{Nanoparticle Synthesis and Characterization}

All chemicals were purchased from Sigma-Aldrich (St. Louis, MO) and used directly unless otherwise noted. Gold nanostars were synthesized following our seed-mediated method, which has been previously described.[29] In short, 12-nm gold nanosphere seeds were synthesized via the reduction of $\mathrm{HAuCl}_{4}(100 \mathrm{ml}, 1 \mathrm{mM})$ by trisodium citrate $(15 \mathrm{ml}$, $1 \% \mathrm{w} / \mathrm{v})$. $60-\mathrm{nm}$ GNS were synthesized by rapidly and simultaneously mixing $\mathrm{AgNO}_{3}(1 \mathrm{ml}, 3 \mathrm{mM})$ and ascorbic acid $(500 \mu \mathrm{l}, 0.1 \mathrm{M})$ with $100 \mathrm{ml}$ of a solution containing $0.25 \mathrm{mM} \mathrm{HAuCl}_{4}, 1 \mathrm{mM} \mathrm{HCl}$, and $1 \mathrm{ml}$ of the gold nanosphere seeds synthesized in the previ- 
ous step. 30-nm GNS were prepared with the same method except the volume of gold nanosphere seeds added was increased to $2 \mathrm{ml}$. Thiolated polyethylene glycol (PEG, $6 \mathrm{kDa}$ ) polymer was added in large excess (50,000 PEG molecules per nanoparticle) to passivate the nanoparticle surface. Raman reporter, p-mercaptobenzoic acid (pMBA, $10 \mu \mathrm{M})$, was also added to the obtained gold nanoparticle solution. After 8 hours incubation at room temperature, the nanoparticle solution was pelleted and resuspended in water. All water used in this study was ultrapure (18 M $\Omega-\mathrm{cm})$.

Synthesized nanoparticles were characterized by transmission electron microscopy (TEM) using $160 \mathrm{kV}$ acceleration voltage (Tecnai $\mathrm{G}^{2}$ Twin, FEI, Hillsboro, OR) and by VIS-NIR spectrophotometry (UV-3600, Shimadzu, Japan) at the Shared Materials Instrumentation Facility of Duke University (https://smif.lab.duke.edu/). The TEM sample was prepared by drying diluted nanoparticle solutions on a formvar/carbon-coated copper grid. The nanoparticles' concentration, hydrodynamic size, and $\zeta$-potential were measured by nanoparticle tracking analysis (NS 500, Nanosight, UK) and dynamic light scattering (Zetasizer Nanoseries, Malvern, UK) at $25^{\circ} \mathrm{C}$.

To investigate the elemental composition of the synthesized nanoparticles, 30-nm and 60-nm GNS were dissolved in aqua regia, and then gold and silver concentrations were measured with atomic absorption spectroscopy (Atomic Absorption Spectrometer 3100, Perkin Elmer, Waltham, MA).

\section{Mouse Models}

All animal studies were performed in accordance with protocols approved by the Duke University Institutional Animal Care and Use Committee. For the radiolabeled GNS biodistribution study, mice with xenograft sarcomas were prepared by injecting mouse sarcoma cells in $150 \mu \mathrm{l}$ of phosphate buffered saline (PBS) solution into the leg muscle of nude mice. Sarcoma cell lines for intramuscular injection were prepared from mouse primary sarcomas, which were homogenized and enzymatically digested as described previously.[33] The obtained cells were cultured in Dulbecco's modified Eagle's medium (DMEM) supplemented with $10 \%$ fetal bovine serum (FBS) for 5 to 8 passages before implantation. The mice with xenograft tumors were used for the biodistribution study when the tumors reached $8-\mathrm{mm}$ diameter. For the in vivo CT imaging and PTT studies, primary soft-tissue sarcomas were generated in the right hind leg of LSL-Kras ${ }^{G 12 D}$; p53 FL/FL compound mutant mice between 6 and 10 weeks of age via intramuscular injection of an adenovirus expressing Cre recombinase (University of Iowa, Carver College of Medicine Viral Vector Core Facility).[34] LSL-Kras ${ }^{G 12 D}$ and $p 53^{F L / F L}$ mice have been described in previous studies.[35, 36]

\section{In Vivo Biodistribution with Radiolabeled Nanostars}

No-carrier-added $\mathrm{Na}^{131} \mathrm{I}$ in $0.1 \mathrm{M} \mathrm{NaOH}$ with a specific activity of $1200 \mathrm{Ci} / \mathrm{mmol}$ was purchased from Perkin-Elmer Life and Analytical Sciences (Boston, MA). Concentrated PEGylated GNS were incubated with ${ }^{131} \mathrm{I}(5 \mu \mathrm{Ci}$ per $200 \mu \mathrm{g}$ gold) in PBS for 2 hours at room temperature. Unbound ${ }^{131} \mathrm{I}$ was removed by pelleting and resuspending the nanoparticles in sterile PBS. The retention of ${ }^{131} \mathrm{I}$ on the GNS surface was investigated in PBS with $4 \%$ albumin. After 96 hours incubation at $37^{\circ} \mathrm{C}$, the solution was centrifuged and the radioactivity of the supernatant and pellet was measured to determine the percentage of ${ }^{131}$ I remaining on the GNS surface. Freshly radiolabeled GNS were used for biodistribution studies. Twelve mice with xenograft sarcomas were randomly divided into 3 groups ( $\mathrm{n}=4$ in each group). Nanoparticles in PBS were injected intravenously via the tail vein. For the first group, $100 \mu \mathrm{l}$ of radiolabeled 30-nm GNS (50 $\mu \mathrm{g}$ gold) was injected. For the second group, $100 \mu \mathrm{l}$ of radiolabeled 30-nm GNS (200 $\mu$ g gold) was injected. For the third group, $100 \mu \mathrm{l}$ of radiolabeled 60-nm GNS (200 $\mu \mathrm{g}$ gold) was used. The injection standard (5\%) for each dose was prepared by diluting $100 \mu \mathrm{l}$ of radiolabeled GNS solution to $2 \mathrm{ml}$ with water. Mice were sacrificed 48 hours after injection and organs of interest were harvested for radioactivity measurement. The radioactivity of each organ and internal standard was measured with an automated gamma counter (LKB 1282, Wallac, Finland), and the measured results were used to calculate percentage of injected dose per gram tissue (\% ID/g) for each organ of interest.

\section{In Vivo Tumor CT Imaging}

Mice with primary sarcomas were used for CT and optical imaging at 12 weeks post-Adeno-Cre infection, at which time point large sarcomas $(\sim 0.5-1 \mathrm{~cm}$ diameter) were present in each mouse. A total of 3 mice were used for the CT and optical imaging study. Longitudinal dual energy (DE) micro-CT imaging was done in all animals. DE imaging was used to accurately quantify in vivo concentrations of gold dynamically. The gold nanoparticle contrast agent (12-nm nanospheres, 30-nm GNS or 60-nm GNS) was injected intravenously through the tail vein at a gold dose of $20 \mathrm{mg} / 25 \mathrm{~g}$ body weight and post-injection DE CT scans were immediately acquired. Additional DE CT scans were done at 24 hours and 72 hours 
post-injection. Following the final scan, the mice were euthanized and tissues were harvested, as described below. A custom-built dual-source micro-CT imaging system was used for the study.[37] Animal monitoring and CT data acquisition were performed and raw CT images processed, as described previously.[38] In short, two acquired datasets $(80 \mathrm{kVp}$ and $40 \mathrm{kVp}$ energies) were reconstructed using the Feldkamp algorithm, co-registered, and smoothed using joint bilateral filtration. Dual energy decomposition was performed by fitting the voxel data to a CT sensitivity matrix derived from gold concentration standards, which we have previously validated in vivo.[39] The result of the dual energy decomposition was a gold concentration map, in which intensity values represent the calculated concentration of gold within each voxel. CT images were manually segmented using Avizo (Visualization Sciences Group, FEI, Hillsboro, OR). Following segmentation, gold concentrations were measured in each region of interest by calculating the average value of the gold maps over the entire region of interest.

\section{SERS Measurement}

In vivo SERS spectra were recorded with a PIXIS: 100BR_eXcelon CCD mounted to an Acton LS-785 spectrograph (1200 grooves/mm grating), controlled by LightField software (Princeton Instruments, Trenton, NJ). A 785-nm diode laser was fiber-coupled to the excitation fiber of an InPhotonics Raman probe (Norwood, MA) and the collection fiber of the RamanProbe was coupled to the entrance slit of the LS-785 spectrograph. SERS spectra were recorded for mice in the CT study immediately after euthanasia. Spectra were recorded for all mice through the skin over the tumor and over the leg muscle on the contralateral side as a control.

\section{Tissue Staining}

Frozen tumor sections ( $8 \mu \mathrm{m}$ thick) were immunostained for the endothelial cell marker CD31. Prior to staining, sections were fixed with $4 \%$ paraformaldehyde, rinsed with PBS, and blocked with $10 \%$ FBS in PBS for one hour. The sections were then incubated with the primary antibody (rat anti-mouse CD31, BD Pharmingen, Franklin Lakes, NJ) diluted 1:250 in blocking buffer for 2 hours at room temperature. The slides were rinsed three times with PBS for 10 minutes to remove unbound primary antibody, after which the secondary antibody (Alexa Fluor 488-conjugated donkey anti-rat IgG, Invitrogen, Carlsbad, CA) diluted 1:500 in blocking buffer was added and incubated for 1 hour in the dark. Nuclei were counterstained with 4', 6-diamidino-2phenylindole (DAPI). A few sections were also used for hematoxylin and eosin (H\&E) staining. For H\&E staining, samples were stained with hematoxylin, rinsed with water and ethanol, stained with eosin $Y$, then dehydrated with ethanol and xylene, and mounted for microscopy.

\section{Two-Photon Luminescence Imaging}

Two-photon luminescence imaging on immunostained tumor sections was performed using an Olympus FV 1000 multiphoton microscope (Olympus America, Center Valley, PA). The pulsed laser used was a Ti:Sapphire laser (Chameleon Vision II; Coherent, Santa Clara, CA) with tunable range of 680-1080 nm, 140 fsec pulse width, and $80 \mathrm{MHz}$ repetition rate. The laser beam (800 $\mathrm{nm}$ wavelength) was focused with a water-immersion objective with 25X 1.05 NA (Olympus America, Center Valley, PA). Images were acquired for each channel (red, green, and blue filter cubes with PMTs) and were merged for the final TPL images.

\section{Dark Field and Fluorescence Imaging}

Immunostained sections were imaged using a Zeiss Axiovert 135 inverted microscope (Carl Zeiss Inc., Thornwood, NY) with a Cytoviva darkfield condenser and dual mode fluorescence module (Cytoviva, Auburn, AL) fitted with a triple bandpass emission filter. All images were acquired using a 20x lens. Each tumor was imaged using both fluorescence and darkfield modes. For darkfield imaging, indirect sample illumination enables image production from light scattered by the samples. Gold nanoparticles are readily detected by darkfield microscopy due to their increased scattering properties relative to the surrounding tissue. Combined darkfield and fluorescent images of each tumor were produced by first thresholding the darkfield images to include only the bright gold nanoparticles. The fluorescence images were then separated into individual color channels in ImageJ (http://imagej.nih.gov/), each of which was thresholded to remove overlapping signals. The resulting images were overlaid, which allowed visualization of the spatial relationship between the gold nanoparticles and the vasculature and nuclei within the tumors.

\section{In Vitro Photothermal Heating Characteriza- tion}

The photothermal effect for 30-nm GNS at different concentrations and laser power densities was investigated by irradiating $0.8 \mathrm{~mL}$ of GNS solution in a plastic vial for 20 minutes with a $980 \mathrm{~nm}$ continuous wavelength laser. A thermocouple temperature probe (VWR, Randnor, PA) was inserted into the solution and used to measure temperature during laser irradiation. The GNS stability during laser irradiation was 
tested by repeating three cycles of laser irradiation on and off for 10 minutes. Calculation of photothermal conversion efficiency was performed following a previously described method.[40, 41] To summarize, 1.5 $\mathrm{ml}$ solutions of PBS, 30-nm GNS, 60-nm GNS, and silica-core gold nanoshells $(120 \mathrm{~nm}$ core, $15 \mathrm{~nm}$ shell, courtesy of Dr. Jennifer L. West Lab, Duke University) at equal optical densities $(0.5,1$, and 1.5$)$ were placed in a quartz cuvette and heated using an 808-nm diode laser operating at a power of $1 \mathrm{~W}$. The laser fiber optic probe was oriented horizontally and was located 0.5 $\mathrm{cm}$ from the base of the cuvette. Solution temperature was monitored using an immersed thermocouple temperature probe positioned $1 \mathrm{~cm}$ above the laser path. The top of the cuvette was covered by insulating foam to reduce heat loss from the surface of the solution. After reaching a temperature plateau at 25 minutes, the laser was turned off and temperature recording was continued until the solution returned to room temperature. From the calculated exponential fit of the heating and cooling curves, we determined the characteristic time constant and heat transfer coefficient of the system. We then used this information, along with the system heat capacity, laser intensity, and solution optical density to calculate the photothermal conversion efficiency for each nanoparticle type. This conversion efficiency is defined as the fraction of absorbed laser power that is converted to heat by the nanoparticles. For a detailed description of the photothermal conversion efficiency calculations, refer to $[40,41]$.

\section{In Vivo Photothermal Therapy}

A 980-nm continuous-wavelength laser was used for the in vivo photothermal therapy. The maximum permissible exposure (MPE) for this wavelength is calculated to be $0.72 \mathrm{~W} / \mathrm{cm}^{2}$ according to American National Standards Institute (ANSI) laser safety guide. The tumor surface temperature during laser irradiation was continuously and remotely monitored with an infrared thermal imaging camera (Thermo Tracer TS 7302, NEC, Japan). In addition, a thermocouple temperature probe was also used to measure tumor surface temperature before and after laser irradiation to verify temperature measured from an infrared thermal imaging camera. Two groups of mice with primary sarcomas $(\mathrm{n}=2$ in each group) were used. For the treatment group, $2 \mathrm{mg}$ of 30-nm GNS were injected two days before photothermal therapy, while PBS solution was injected for the control group. The tumors were irradiated at $0.7 \mathrm{~W} / \mathrm{cm}^{2}$ for 10 minutes on both the front and back sides. Afterwards, the mice were monitored and the photothermal therapy effect was evaluated by observing the change in tumor size.

\section{Statistics}

Differences between groups in the biodistribution and photothermal conversion efficiency studies were analyzed using a one-way ANOVA followed by post-hoc testing with the Fisher's PLSD test. All data are represented as the mean \pm standard deviation.

\section{Results}

\section{Nanostar Characterization}

GNS (30-nm and 60-nm) were synthesized via a seed-mediated method, in which gold ions were reduced onto the surface of 12-nm gold nanospheres using ascorbic acid in the presence of $\mathrm{AgNO}_{3}$. This procedure causes asymmetric growth of the gold cores, resulting in the development of multiple gold spikes. TEM images and UV-Vis spectra of the synthesized nanoparticles are shown in Figure 1.
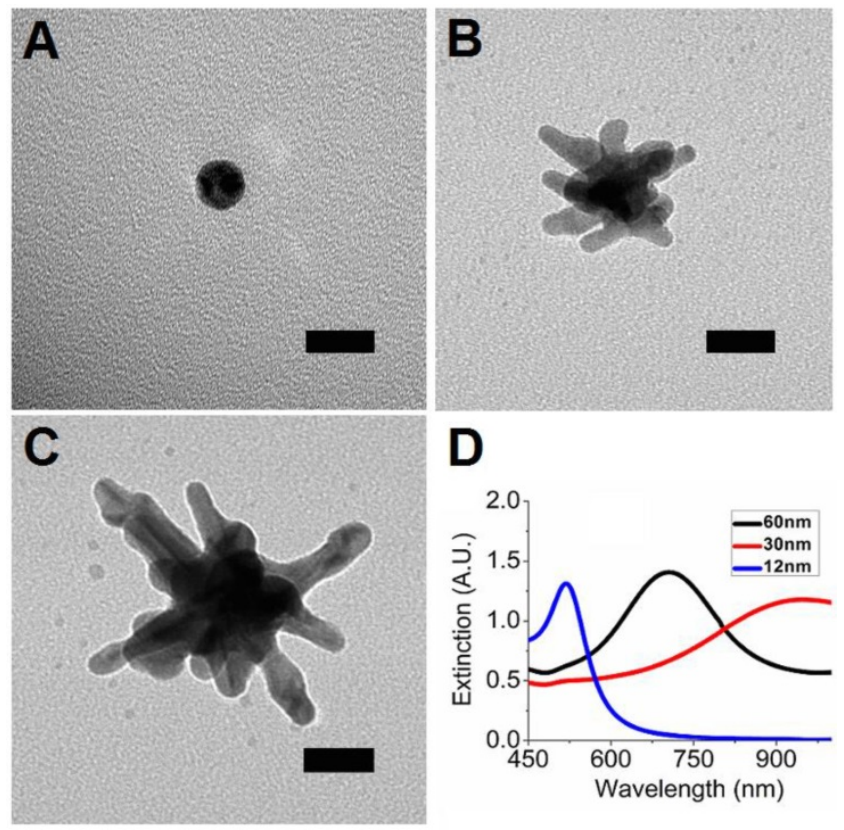

D

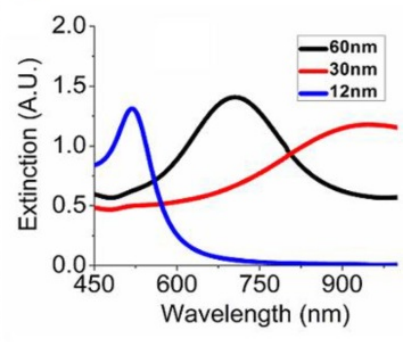

Figure 1. TEM images of 12-nm gold nanospheres (A), 30-nm GNS (B), and 60-nm GNS (C). UV-Vis spectra of 12-nm gold nanospheres, 30-nm GNS, and 60-nm GNS solutions (D). The plasmon peaks are $520 \mathrm{~nm}$ for 12-nm nanospheres $(5 \mathrm{nM}), 945 \mathrm{~nm}$ for 30-nm GNS $(0.2 \mathrm{nM})$, and $706 \mathrm{~nm}$ for $60-\mathrm{nm}$ GNS $(0.1 \mathrm{nM})$, respectively. Scale bar is $20 \mathrm{~nm}$ in all images.

The 12-nm nanospheres have a plasmon peak at $520 \mathrm{~nm}$ while the GNS have peaks at $706 \mathrm{~nm}(60-\mathrm{nm}$ GNS) or $945 \mathrm{~nm}$ (30-nm GNS). Nanoparticle size and $\zeta$-potential measurements are shown in Table 1. The hydrodynamic diameter for each particle type is significantly higher than the physical diameter shown with TEM, reflecting the increase in effective particle size due to PEG coating. The obtained nanoparticles have $\zeta$-potential close to neutral after PEG coating, which demonstrates effective shielding of the GNS surface charge by PEG. This is important for minimizing particle aggregation, plasma protein binding, 
and host nanoparticle recognition. The resulting nanoparticles exhibit no aggregation under physiological salt conditions. AAS measurements showed that 30-nm GNS contain $95.5 \%$ gold and $4.5 \%$ silver by mass while $60-\mathrm{nm}$ GNS contain $96.9 \%$ gold and $3.1 \%$ silver.

Table 1. Nanoparticle size (by TEM and DLS) and $\zeta$-potential of three nanoparticles investigated in this study. TEM nanoparticle diameter was measured end-to-end.

\begin{tabular}{|c|c|c|c|}
\hline TEM diameter & $12 \mathrm{~nm}$ & $30 \mathrm{~nm}$ & $60 \mathrm{~nm}$ \\
\hline Hydrodynamic diameter & $38 \mathrm{~nm}$ & $56 \mathrm{~nm}$ & $73 \mathrm{~nm}$ \\
\hline Polydispersity Index & 0.14 & 0.30 & 0.25 \\
\hline$\zeta$-potential & $-7.1 \mathrm{mV}$ & $-3.1 \mathrm{mV}$ & $-5.7 \mathrm{mV}$ \\
\hline
\end{tabular}

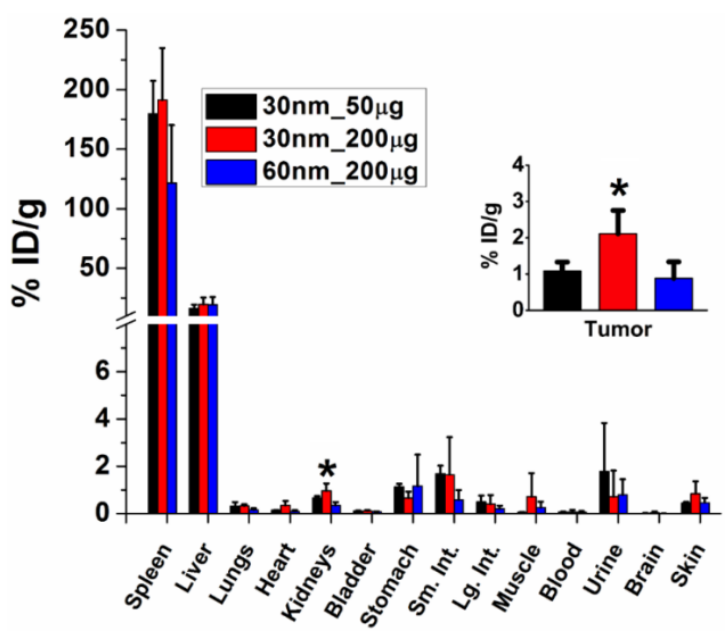

Figure 2. Biodistribution (\%ID/g) of GNS with different particle sizes and injection doses 48 hours after tail vein injection. Four mice with xenograft sarcomas were used for each group. Smaller nanostars with higher injection dose have higher tumor uptake. The \% $\mathrm{ID} / \mathrm{g}$ is defined as percentage of total injection dose per gram tissue weight. Error bars show standard deviation. The asterisk represents a statistically significant difference from the other two groups $(p<0.05)$.

\section{Nanostar In Vivo Biodistribution}

A nanoparticle's in vivo biodistribution and tumor uptake are of critical importance for its use in cancer imaging and treatment. Radioisotope labeling provides a sensitive and quantitative method to investigate nanoparticle biodistribution. For the biodistribution study, GNS were labeled with ${ }^{131} \mathrm{I}$, which has a half-life of $\sim 8$ days. Iodine radioisotopes show high labeling efficiency (more than 99\%), as well as good stability on gold nanoparticles due to strong iodine-gold interaction.[42] The retention of 131I on the GNS surface was investigated in PBS with $4 \%$ albumin and results showed that $99 \%$ of $131 \mathrm{I}$ remained on the nanoprobes after incubation at $37^{\circ} \mathrm{C}$ for 72 hours.

Radiolabelled GNS (30 nm and $60 \mathrm{~nm}$ ) were injected intravenously into mice with xenograft sarcomas, and radioactivity uptake in tumor and other organs of interest was measured after 48 hours; results are shown in Figure 2. Tumor uptake of 30-nm GNS was higher $(2.11 \pm 0.64 \% \mathrm{ID} / \mathrm{g})$ than that of $60-\mathrm{nm}$ GNS $(0.88 \pm 0.46 \% \mathrm{ID} / \mathrm{g})$ with the same injection dose of gold $(200 \mu \mathrm{g})$. In addition, 30-nm GNS showed a higher tumor uptake with a 200- $\mu$ g injection dose $(2.11 \pm 0.64 \% \mathrm{ID} / \mathrm{g})$ than with $50-\mu \mathrm{g}$ injection dose $(1.08 \pm 0.25 \% \mathrm{ID} / \mathrm{g})$. This difference was found to be statistically significant. The reticuloendothelial system (RES), including liver and spleen, demonstrated high uptake of nanoparticles, which is consistent with previous studies.[43] Relatively low uptake levels of nanoparticles were seen in other non-target organs.

\section{Tumor CT Imaging}

In order to further investigate gold nanoparticles' dynamic biodistribution and demonstrate real-time monitoring of intratumoral accumulation, we performed dual-energy CT imaging on mice with primary sarcomas 30 minutes, 24 hours, and 72 hours after gold nanoparticles injection. Mice with primary sarcomas are immunocompetent and may more accurately reflect the tumor microenvironment and response to therapy of human tumors compared to xenograft models.[44-46] The longitudinal dual-energy CT imaging showed gold nanoparticle distribution at various time points (Figure 3). The CT images at 30 minutes clearly show gold nanoparticles localized to blood vessels. It appears that the majority of blood vessels in the tumor region were located on the outside of the tumor, so perfusion to the core of the tumor may be limited. At the 24-hour and 72-hour time points, there was nanoparticle accumulation in the tumors. Concentrations of gold in the blood, tumors, and other organs calculated from each dual-energy CT scan are shown in the Supplementary Figure S1. These results are consistent with the results obtained with radiolabelled GNS, showing significant RES accumulation and tumor uptake, as well as decreasing concentrations of gold within the blood over time. The most notable difference between these two biodistribution studies is that the concentration of gold in the tumor relative to the liver and spleen was much higher in the CT study than in the radiolabeled study. This is most likely due to the much higher dose of gold injected for the CT study. The RES might be saturated with a high injection dose, which would give the nanoparticles a longer circulation time. It is also possible that the tumor nanoparticle uptake differs between the primary tumor and xenograft mouse models. Blood residence time for each nanoparticle type was approximated using the measured gold concentrations in the blood at 0,24 , and 72 hours post-injection. 12-nm nanospheres had a blood half-life of approximately 26 hours; $30-n m$ GNS had a half-life of 33 hours; and 60-nm GNS had a half-life of 
27 hours, which is consistent with residence times we have seen in previous gold nanoparticle studies.[39] These CT results also show a comparison of the concentrations of gold within the tumor rim and tumor core. In all three cases, there was high gold concentration in the tumor rim and negligible gold concentration in the tumor core. CT slices through the tumors demonstrate this heterogeneous intratumoral distribution (Figure 4). The gold concentration at the rim is much higher than that in the center and is not evenly distributed around the tumor rim. The low concentration at the center may reflect poor perfusion in the tumor core. This is supported by the H\&E images (Supplementary Figure S2), which show tumor necrosis at the core, but viable tumor near the margins. Additionally, the 12-nm nanospheres showed much more skin accumulation around the tumor site than the larger GNS. Images of the gross primary sarcomas before extraction are shown in Supplementary Figure S3. They show that the surface of the sarcoma is black in color due to high GNS accumulation, consistent with the CT cross-sectional images. There is also a clearly visible color boundary between tumor and normal muscle due to differential GNS uptake, which indicates that GNS could potentially be used as an intraoperative imaging contrast agent during cancer surgery.

\section{Optical Imaging of Nanoparticle Distribution in Tumors}

GNS have extremely strong two-photon luminescence and can be used to monitor nanoparticle distribution at the cellular level without dye labeling. GNS also have a large enough scattering cross-section to allow imaging by darkfield microscopy, in which only the light scattered from indirect illumination of the sample is detected. After CT imaging, tumors were sectioned, immunostained and imaged using two-photon microscopy, fluorescence microscopy, and darkfield microscopy. As shown in Figure 5, both the 30-nm and 60-nm GNS show extremely strong two-photon luminescence. The emitted light spans the visible spectrum, so the nanoparticles appear bright white. It is noteworthy that $12-\mathrm{nm}$ gold nanospheres showed no detectable TPL signal. All three nanoparticle types were visible by darkfield microscopy, but the GNS were much less easily visualized with this modality compared to TPL imaging. Both imaging methods were able to show tissue-level distribution of nanoparticles within the tumor. The $12-\mathrm{nm}$ nanospheres and 30-nm GNS nanoprobes penetrate more deeply into the tissue than 60-nm GNS after leaking through tumor vasculature. The 12-nm nanospheres and 30-nm GNS have a fairly even distribution in tumor tissue, while the larger 60-nm GNS were restricted primarily to the perivascular space.

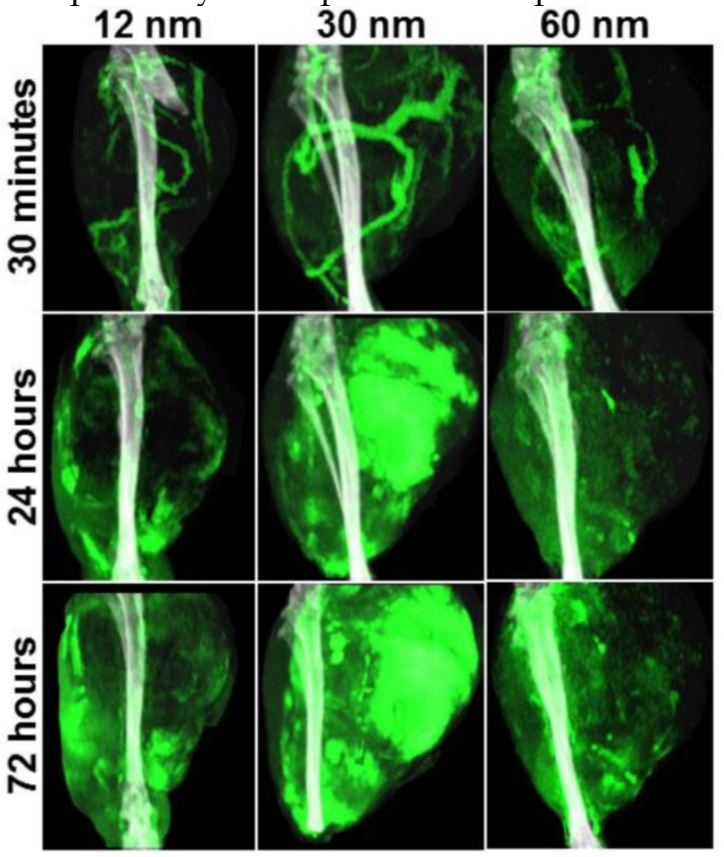

Figure 3. Maximum intensity projection CT images of hind leg primary sarcomas at 30 minutes, 24 hours, and 72 hours after gold nanoparticle injection (12-nm nanospheres, 30-nm and 60-nm GNS). Gold concentrations are shown in green (windowed from 2 to $10 \mathrm{mg} / \mathrm{ml}$ ), while other tissues are shown in gray scale (windowed from -100 to $5000 \mathrm{HU}$ ). Gold is primarily visualized in the blood vessels immediately after injection and in the tissues in the delayed scans.

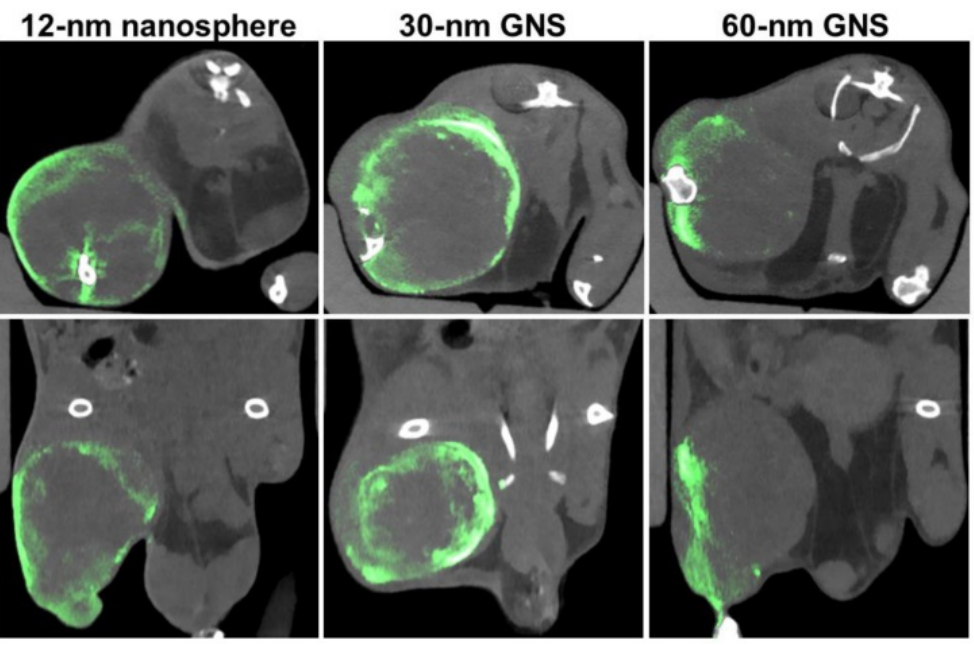

Figure 4. Dual-energy CT axial slices (top) and coronal slices (bottom) in primary sarcomas 72 hours post-injection with $12-\mathrm{nm}$ gold nanospheres, 30-nm GNS, and 60-nm GNS. Gold is shown in green (windowed from 2.5 to $8 \mathrm{mg} / \mathrm{ml}$ ), while all other tissues are shown in gray scale (windowed from -500 to $1200 \mathrm{HU}$ ). 


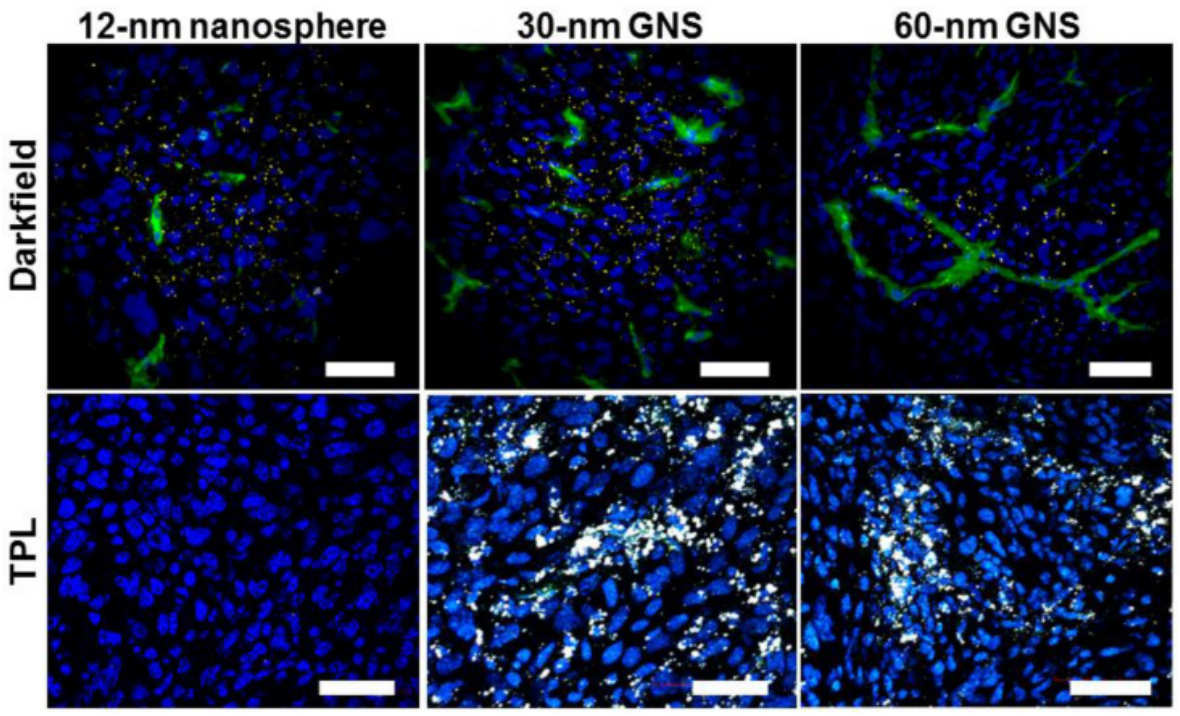

Figure 5. High-resolution darkfield/fluorescence microscopy (top) and two-photon luminescence (TPL) microscopy (bottom) for gold nanoparticle tracking in tumors after leaking through vasculature. 12-nm gold nanospheres were undetectable with TPL imaging in this study, but all nanoparticles were visible darkfield. Gold nanoparticles with smaller size penetrate more deeply into tumor interstitial space. Scale bar, $50 \mu \mathrm{m}$. Blue, DAPI; green, CD31; gold nanostars appear yellow in darkfield images and white in TPL images.

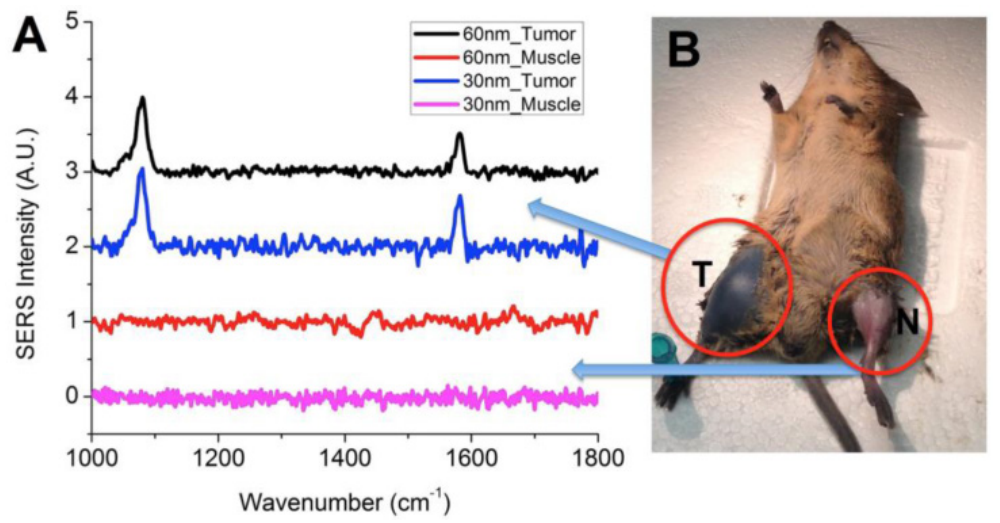

Figure 6. (A) In vivo SERS spectra of 30-nm and 60-nm GNS nanoprobes with pMBA reporter. Unique SERS peaks can be detected at 1067 and $1588 \mathrm{~cm}-1$ in the tumor, but not in the normal muscle. Baselines are artificially offset to visualize each spectrum individually. (B) Mouse with primary sarcomas 3 days after 30-nm GNS injection. Significant GNS accumulation can be seen in the tumor $(\mathrm{T})$, but not in the normal leg muscle of the contralateral leg $(\mathrm{N})$.

\section{GNS Nanoprobe for in vivo SERS Measure- ments}

Gold nanostars exhibit very intense SERS signal due to strong local field enhancement at the tips of the nanostar spikes. Our previous study showed that GNS SERS enhancement is more than two orders of magnitude higher than that of gold nanospheres.[31] Following CT imaging, the SERS signal from the GNS that accumulated within each tumor and contralateral leg muscle was measured. Figure 6A shows the SERS spectrum for 30-nm GNS and 60-nm GNS in the sarcoma and normal muscle. The GNS were labeled with pMBA, a Raman reporter. The characteristic SERS peaks of pMBA on GNS at 1067 and $1588 \mathrm{~cm}^{-1}$ were detected in the tumor, but not in the contralateral leg muscle, which shows that SERS has the capability to differentiate tumor from normal muscle. The SERS spectra for Raman reporter pMBA from 30-nm and 60-nm GNS are nearly identical. Those two major peaks were assigned to the stretching vibrational mode of the benzene ring in pMBA based on previous combined theoretical and experimental investigations. $[47,48]$

\section{GNS Nanoprobe for Photothermal Therapy}

In addition to its use as an imaging and tumor detection agent, the developed multifunctional GNS probe can also be used for photothermal therapy. Because the 30-nm GNS accumulate more in tumors and have deeper tissue penetration than the 60-nm GNS, we selected 30-nm GNS to investigate in vivo photothermal therapy. The 30-nm GNS probe was first tested with in vitro experiments, the results of which are shown in Figure 7. After three repetitive laser irradiations $\left(0.8 \mathrm{~W} / \mathrm{cm}^{2}\right)$, there was no decrease in the 
photon-to-heat conversion (Figure 7A). Figures 7B and $7 \mathrm{C}$ demonstrate that equilibrium temperature varies with GNS concentration and laser power. Water (no GNS) increased from room temperature to $\sim 31$ ${ }^{\circ} \mathrm{C}$ after extended laser irradiation, while the $0.5 \mathrm{nM}$ GNS solution increased to $\sim 42{ }^{\circ} \mathrm{C}$ upon continuous irradiation. Equilibrium was reached after about 10 minutes of irradiation at all GNS concentrations. This equilibrium temperature depends strongly on laser power, as shown in Figure 7C. Although increasing laser power clearly led to higher final temperatures, the $0.7 \mathrm{~W} / \mathrm{cm}^{2}$ laser power was chosen for in vivo studies in order to minimize non-target tissue damage and keep exposure levels under the recommended maximum permissible exposure (MPE). We also performed calculations to investigate how efficiently GNS can facilitate photothermal therapy (Supplementary Figure S4). The results suggest that GNS have a high photothermal conversion efficiency and that the temperature at the GNS surface could be high enough to vaporize surrounding water molecules. This finding is supported by the presence of significant condensation on the sides of the cuvette after even short periods of irradiation (with bulk solution temperature $<40^{\circ} \mathrm{C}$ ).

We then performed a direct measurement of photothermal conversion efficiency for the 30-nm and 60-nm GNS, and we compared their efficiency to gold nanoshells, which are one of the most well-studied nanoparticles used for photothermal therapy.[49, 50] Figure 7E shows the temperature profiles for each of these three nanoparticles, with the $30-\mathrm{nm}$ and $60-\mathrm{nm}$ GNS having a much higher equilibrium temperature $\left(42.3^{\circ} \mathrm{C}\right.$ and $\left.41.4{ }^{\circ} \mathrm{C}\right)$ than nanoshells $\left(34.7{ }^{\circ} \mathrm{C}\right)$ at equivalent optical density. The calculated values of photothermal conversion efficiency (Figure 7F) were $94 \%$ for $30-n m$ GNS, $90 \%$ for $60-n m$ GNS, and $61 \%$ for gold nanoshells. The difference in efficiencies between the gold nanoshells and the two sizes of GNS was found to be statistically significant. This demonstrates that GNS have a higher efficiency in converting the delivered laser power into heat.

Following in vitro evaluation, we performed in vivo photothermal therapy on mice bearing primary sarcomas. Figure 8 shows NIR images depicting surface temperature change during the photothermal treatment process and the measured temperature increase of sarcomas with and without GNS over 10 minutes of laser exposure. The tumor temperature is much higher for mice with GNS injection than for mice with PBS injection. The tumor surface temperature with GNS reaches $50{ }^{\circ} \mathrm{C}$ after only 4 minutes of treatment, which is high enough to kill tumor cells.[51] The tumors treated with GNS and laser irradiation regressed to an undetectable size one day after photothermal therapy with GNS, while tumors treated with PBS and laser irradiation continued to grow rapidly. Photographs and x-ray images of these two conditions are shown in Figure 9. The GNS mice exhibited no sign of tumor growth for 7 days following treatment, at which point they were sacrificed. The laser irradiation was confined primarily to the tumor and there was no detectable tissue damage outside of the tumor region. There was some skin burning directly over the tumor surface, but no other adverse effects were observed in these mice. These results provide the proof of principle that the GNS nanoprobe may provide a useful platform for image-guided photothermal therapy, which will be investigated further in future pre-clinical experiments.
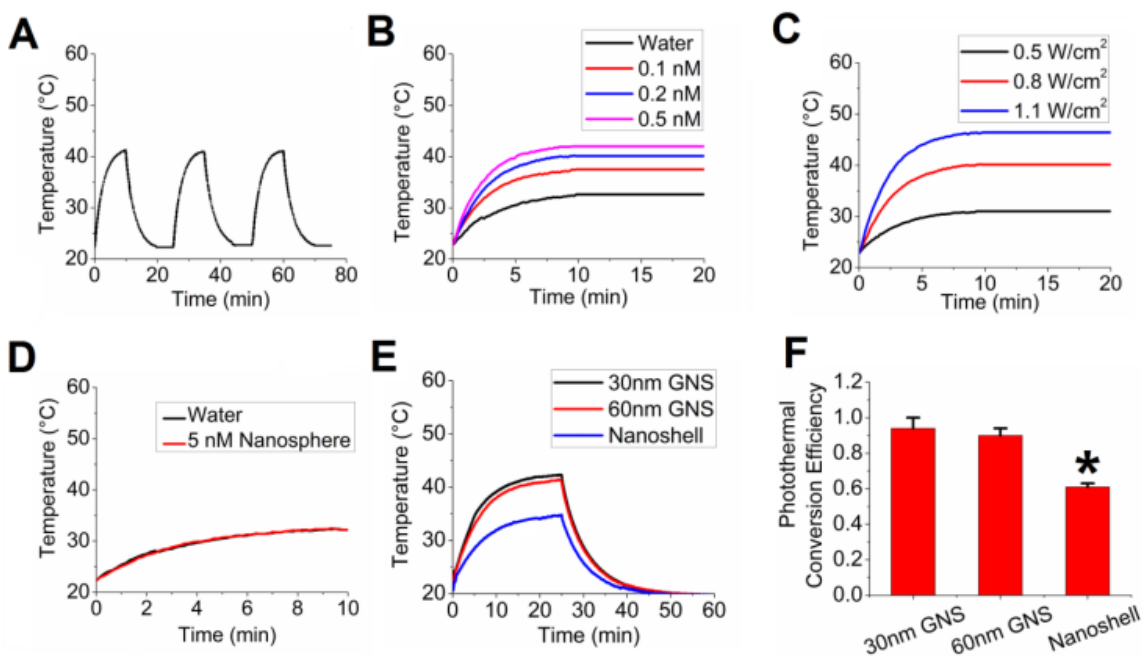

Figure 7. In vitro photothermal heating evaluation. (A) Repetitive photothermal heating $\left(0.2 \mathrm{nM} 30-\mathrm{nm}\right.$ GNS solution) with $0.8 \mathrm{~W} / \mathrm{cm}^{2}$ laser $(980 \mathrm{~nm}$ wavelength) on at 0,25 , and 50 minutes and laser off at 10,35, and 60 minutes. (B) Temperature profile for different GNS concentrations with $0.8 \mathrm{~W} / \mathrm{cm}^{2}$ laser intensity. (C) Temperature profile for $0.2 \mathrm{nM}$ GNS with different laser intensities. (D) Temperature profile for 12 -nm gold nanospheres $(5 \mathrm{nM})$ and water with $0.8 \mathrm{~W} / \mathrm{cm}^{2}$ laser intensity. No photothermal enhancement was observed for 12-nm gold nanospheres compared to water. (E) Temperature profile of 30-nm GNS, 60-nm GNS, and gold nanoshells for photothermal conversion efficiency calculations. (F) Calculated photothermal conversion efficiency for GNS nanoprobes and gold nanoshells as a comparison ( $n=3$ for each group). 


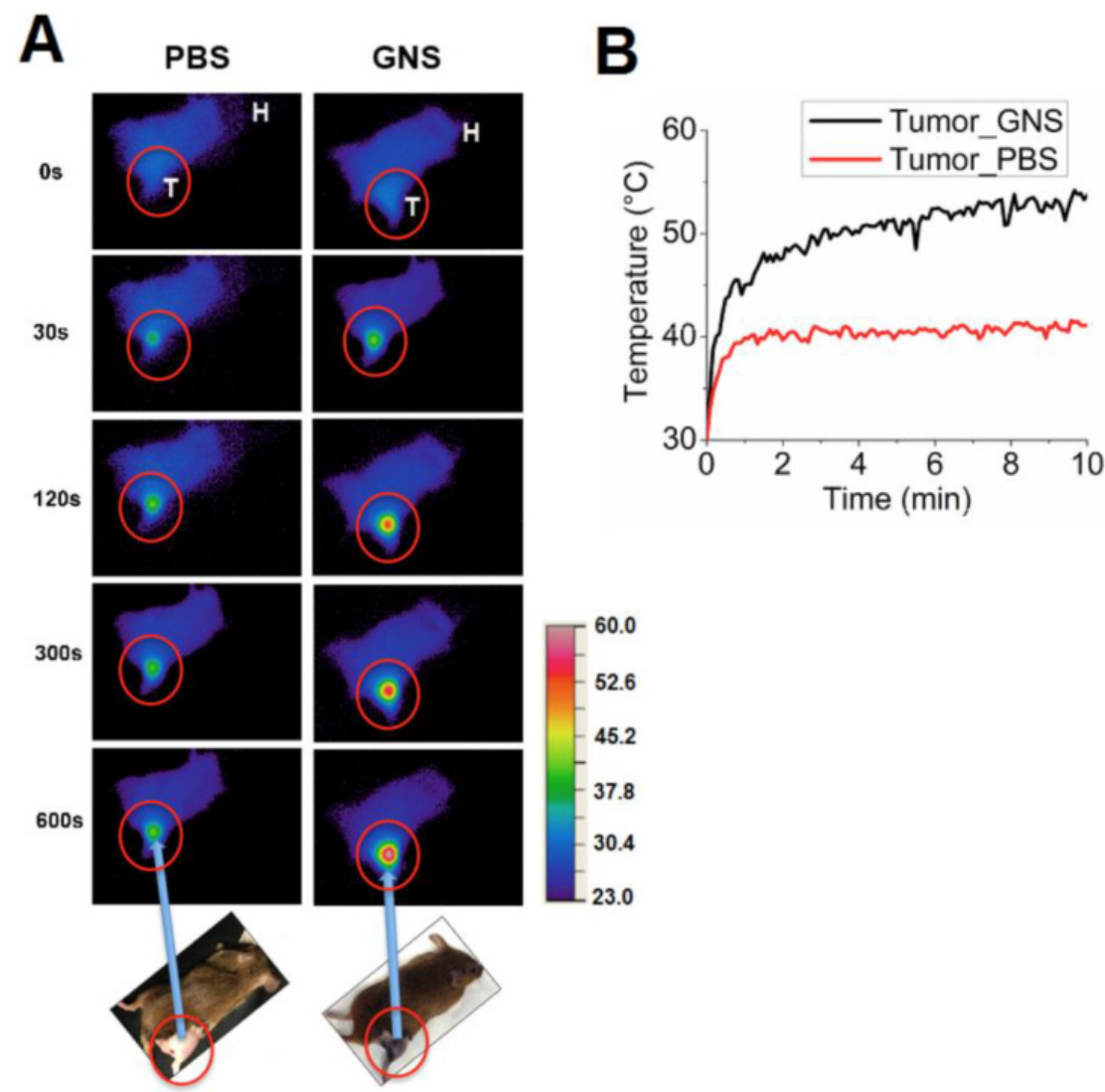

Figure 8. (A) Near-infrared imaging of mouse surface temperature during photothermal treatment of primary sarcomas after intravenous injection of PBS or 30-nm GNS. Each image shows the surface temperature plot of the whole mouse, with the tumor circled in red. T represents the mouse tumor and $\mathrm{H}$ represents the mouse head. The temperature scale bar is between $23^{\circ} \mathrm{C}$ and $60^{\circ} \mathrm{C}$. (B) In vivo profile of the average surface temperature of primary sarcomas with and without injection of GNS measured by a calibrated IR camera.

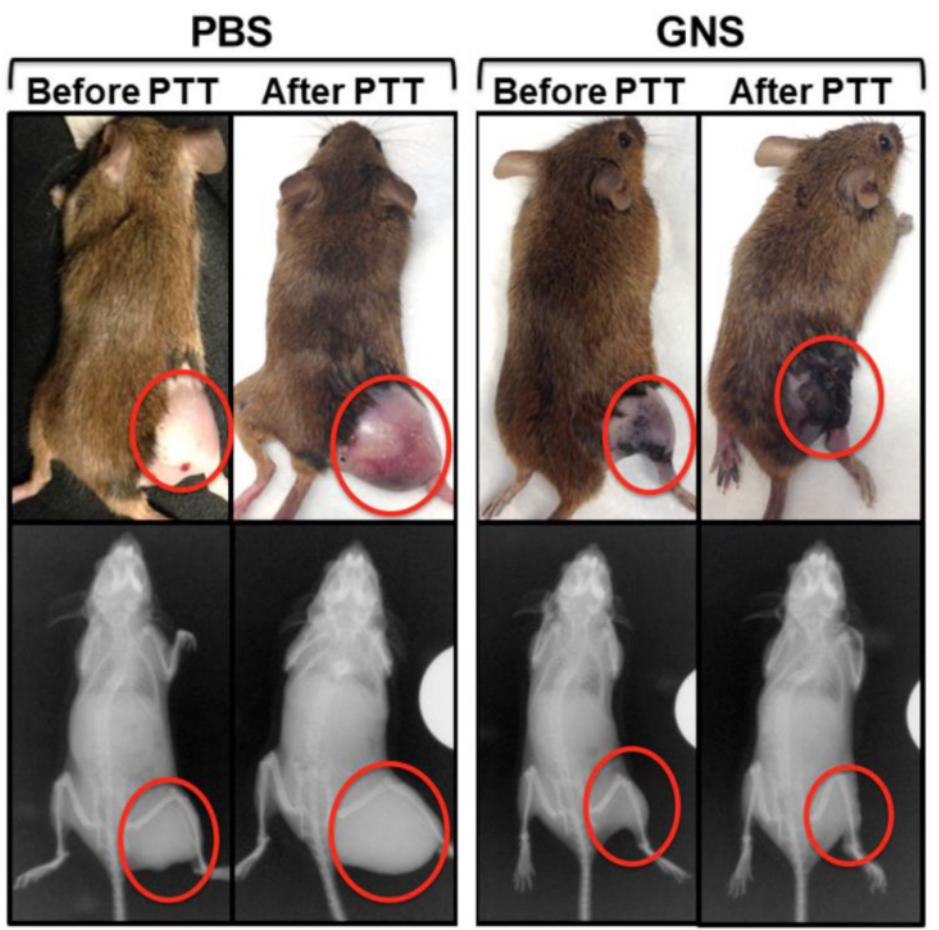

Figure 9. Photographs (top) and x-ray images (bottom) of mice before and after photothermal therapy with tumors circled in red. The control mouse images were taken 7 days after treatment and the images of the mouse with GNS injection were taken 3 days after treatment. Dark discoloration in the tumor region for the GNS mouse is due to nanoparticle accumulation in the underlying tumor. X-ray images show a clear decrease in tumor bulk for the mouse with GNS injection, but a significant increase in tumor size for the mouse with PBS injection. Similar results were obtained for the second mouse tested in each group. 


\section{Discussion and Conclusion}

We have shown in this study that our unique surfactant-free GNS can be effectively used for multimodal in vivo imaging and therapy of cancer in a laboratory animal model. We have demonstrated that (1) GNS can be radiolabelled for particle tracking and biodistribution analysis; (2) they are an effective CT contrast agent that can be monitored dynamically and quantified using dual energy CT; (3) they have high SERS signal; (4) they exhibit intense two-photon luminescence for nanoparticle detection; and (5) they have high photothermal conversion efficiency, which was utilized for tumor photothermal ablation in a proof-of-principle demonstration.

Although other gold nanoparticles have been used for imaging and therapy, these GNS exhibit unique properties including tip-enhanced plasmonics, which provide significant advantages. Not only do GNS have a tunable plasmon resonance peak in the NIR region, but they also have multiple sharp spikes, which generate strong local field enhancement for SERS. The narrow spikes provide a very large absorption cross-section for both TPL imaging and photon-to-heat conversion for PTT. Our surfactant-free synthesis method produces GNS with high biocompatibility and low toxicity, which allows for successful in vivo application of these nanoparticles. This is the first in-depth in vivo demonstration of these novel GNS, and our results suggest that GNS have great promise for further in vivo applications.

The multifunctional GNS nanoprobe has the potential to be used for tumor detection and margin delineation using SERS. Our group has been working on SERS detection for more than two decades.[52-55] SERS takes advantage of surface plasmon resonance and narrow Raman peaks to provide a sensing method with high sensitivity and specificity. GNS are particularly well-suited for SERS sensing due to the strong local field enhancement at the tip of each spike, which greatly increases the SERS signal. In this study, we demonstrated the ability to detect a strong in vivo SERS signal in sarcomas through the intact skin for both 30-nm and 60-nm GNS. A recent study applied SERS imaging with gold nanospheres to identify microscopic glioblastoma extent in a mouse model, and the results demonstrated that the resection under SERS guidance is more accurate than traditional method with white light illumination.[56] The GNS nanoprobes developed in this study have strong SERS enhancement without aggregation (more than 100 times stronger than gold nanospheres),[31] which could be used to significantly improve this SERS-guided surgical technique by detecting tumor margins and residual tumor cells in the surgical bed with high sensitivity. Our future studies will further explore this exciting application for GNS.

The PTT results show a successful proof-of-principle demonstration of GNS-mediated photothermal ablation. In vitro tests showed highly efficient heating even at relatively low laser power. The GNS photothermal conversion efficiency (90-94\%) was significantly higher than that calculated for gold nanoshells (61\%), a nanoparticle commonly used for in vivo photothermal therapy.[49, 50] It should be mentioned that our calculated value for gold nanoshells is somewhat higher than values previously reported.[40,57] The absorption efficiency of nanoshells varies significantly depending on both the core and total diameter of the gold nanoshells. Therefore, it is expected that the calculated conversion efficiency will vary from one formulation to another. In addition, our experimental setup might also yield slightly different results from others in the literature. Although our calculated conversion efficiency values may be high compared to previous reports, the important result from this experiment is the direct comparison between GNS and gold nanoshells, which shows that the GNS have significantly higher conversion efficiency than the commonly-used gold nanoshells. This difference is consistent with prior theoretical calculations, which have shown that GNS have a very high absorption-to-scattering ratio relative to other gold nanoparticle shapes (including gold nanoshells).[30] Because absorption contributes more to photothermal conversion than scattering, heating efficiency tends to correlate well with the absorption-to-scattering ratio. Our theoretical calculations have shown that GNS have the highest absorption-to-scattering ratio of the commonly used plasmonics gold nanoparticles, which is consistent with the very high conversion efficiency seen in this study. Such high conversion efficiency is ideal for in vivo applications, because the conversion efficiency is a measure of how much of the incoming laser light gets converted to heat. Nanoparticles with higher efficiency are capable of achieving higher local temperatures at a given laser power or can use lower laser power to minimize off-target tissue heating. Furthermore, 30-nm GNS also have a much smaller size than that of gold nanoshells, which are typically larger than $100 \mathrm{~nm}$. The small size of GNS would help them leak through tumor vasculature by the enhanced permeability and retention (EPR) effect and penetrate more deeply into tumor interstitial space, making photothermal heating more even and effective for treating tumor cells. Additionally, GNS showed no decrease in effectiveness after multiple rounds of photothermal heating. Despite being heated to very high temperatures, GNS maintain their structure and 
photon-to-heat conversion efficiency, which makes it possible to use GNS for prolonged therapy or for multiple therapy sessions over the course of several days or weeks. Because GNS remain trapped in the tumor tissue by the EPR effect, such longitudinal treatment regimens using only a single injection are highly practical.

The in vivo PTT results indicate that a single 20-minute laser treatment could be sufficient to ablate an otherwise highly-aggressive primary soft-tissue sarcoma. The GNS-treated mice exhibited no tumor recurrence for 7 days, while the tumors in PBS-treated mice continued to grow rapidly. It should be mentioned that the laser irradiation caused some skin burning directly over the tumor in this study, which could be avoided by lowering laser intensity and/or changing laser wavelength in future studies. In this study, a 980-nm laser was used to match the 945-nm plasmon peak of the $30-\mathrm{nm}$ GNS probe. However, there is moderate absorption of $980-\mathrm{nm}$ light by water, which is likely why we noticed some mild damage due to non-specific heating (skin burns) in this experiment. If we tune the GNS plasmon peak to lower wavelengths $(\sim 800 \mathrm{~nm})$, we will be able to use a laser wavelength $(808 \mathrm{~nm})$, which will have much less water absorbance. Given the high photothermal conversion efficiency seen in this study, we should also be able to reduce the laser intensity without significantly reducing our treatment efficacy. Together, these steps should significantly decrease any off-target effects of the laser heating in future studies.

In addition to single PTT treatment tumor ablation, repeat therapy could potentially be given using a fractionated regimen of irradiation to kill additional tumor cells without needing to inject any additional nanoparticles. In addition to photothermal therapy, gold nanoparticles can also be applied to enhance radiation therapy.[58] A combined photothermal therapy and radiation therapy enhanced by GNS might further improve treatment efficacy, and allow a lower dose for both treatment modalities to minimize side effects.

Tracking nanoparticles after leaking through tumor blood vasculature is important for both drug delivery and molecular imaging. Although imaging modalities like CT, MRI, and PET can be used to perform whole body scans, they are insufficient to track nanoparticles at the cellular level. Optical imaging with higher spatial resolution can be used to track nanoparticles; however, labeled dyes may dissociate from nanoparticles in the body, which makes imaging characterization error prone. Additionally, traditional fluorophores commonly quench when attached to gold nanoparticles, making fluorescence imaging difficult for such nanoparticles. As a result, a nanoprobe with intrinsic imaging properties would be more suitable. GNS can be directly imaged using both two-photon and darkfield microscopy, as we have demonstrated in this study. Darkfield microscopy can be used for any nanoparticle that has sufficiently large scattering cross-section, with scattering cross-section generally increasing for larger diameter nanoparticles. We were able to visualize both GNS and 12-nm gold nanospheres in tissue sections using darkfield microscopy; however, the sensitivity of darkfield microscopy for the detection of nanoparticles in tissue is fairly low. A single nanoparticle with size less than 50 $\mathrm{nm}$ cannot readily be detected in tissue sections due to the background signal from tissue light scattering. Although we were able to visualize nanoparticles in the darkfield images, the bright spots most likely represent areas where multiple nanoparticles were congregated or aggregated, which increased the scattering intensity above background levels. Two-photon luminescence, on the other hand, has extremely high sensitivity for detecting GNS. The calculated two-photon absorption cross-section for branched GNS is approximately $5 \times 10^{4}$ times higher than for equivalent-sized gold nanospheres, [59] which means that even single GNS appears very bright when imaged with two-photon microscopy. This can be used, as in the present study, to detect tissue distribution of nanoparticles ex vivo. Two-photon microscopy can also be used for in vivo imaging, and has previously been used by our lab to detect GNS within tissue vasculature and penetration through the blood-brain barrier.[13, 29, 30]

Two-photon and darkfield microscopy of tumor sections showed similar results in this study. The 12-nm nanospheres were undetectable using two-photon microscopy, but showed widespread distribution within the tumor tissue in the darkfield images. 30-nm GNS showed distribution throughout the tumor tissue, but were more concentrated near the blood vessels than in the interstitial space. $60-\mathrm{nm}$ GNS had much less penetration into the interstitial space, and were detected primarily in the perivascular region. This result was expected, as larger nanoparticles diffuse less into tissues than smaller nanoparticles and tend to remain near the vasculature. For therapeutic efficiency, deeper tumor penetration is desirable, and therefore smaller nanoparticles are ideal. The 30-nm GNS were chosen for the PTT study primarily because of their better tumor penetration when compared with the 60-nm GNS.

The GNS probe has also been successfully used as a highly-enhancing contrast agent for CT imaging. $\mathrm{CT}$ is one of the most commonly used imaging modalities in the clinic today and is extremely useful for tumor detection and treatment planning (for both 
surgery and radiation therapy). Developing a probe that can be used for both CT imaging and therapy is highly advantageous, as that single probe can be used for pre-treatment planning, confirmation of therapeutic delivery to the target, therapy, and monitoring of treatment response. CT imaging also provides non-invasive real time dynamic information, which allows us to accurately determine nanoparticle concentrations in different tissues at any given time. This allows us to acquire full biodistribution data dynamically, without having to sacrifice animals at each time point. It also allows us to visualize which regions of the tumor are well vascularized and which do not experience any nanoparticle delivery. GNS tumor distribution seen in CT can also be used for dose painting strategies with radiation treatment.[43, 60] This information can therefore be very useful in improving our understanding of nanoparticle in vivo performance and for treatment planning.

In vivo biodistribution and tumor uptake are of great importance for nanoparticle studies. Particle size, surface charge, and targeting ligands have all been considered to affect nanoparticle in vivo biodistribution and tumor uptake.[21, 42,61-64] The shape of nanoparticles has also been found to affect circulation time and tumor accumulation.[65] GNS have a unique shape with multiple branches compared to traditional nanospheres. Comparing tumor uptake and intratumoral distribution of nanoparticles with different shapes will enable us to identify the most suitable nanoprobe structure for future contrast agent and drug carrier development. In this study, we found the tumor uptake of the GNS to be 1-2 \%ID/g. A recent in vivo study on ${ }^{198} \mathrm{Au}$-doped gold nanoparticles with similar PEG coating reported that nanospheres and nanodisks have much more tumor uptake than that of nanorods and nanocages one day after IV injection.[66] The tumor uptake of different shaped nanoparticles was reported to be $23.2 \% \mathrm{ID} / \mathrm{g}$ (nanospheres), $2.0 \% \mathrm{ID} / \mathrm{g}$ (nanorods), $7.5 \% \quad \% \mathrm{ID} / \mathrm{g}$ (nanocages) and $4.9 \% \mathrm{ID} / \mathrm{g}$ (nanodisks) at the end of $24 \mathrm{~h}$. The differences seen in tumor uptake can be attributed not only to differences in shape, but also to differences in tumor type and injection dose. The previous study used EMT6 murine mammary carcinoma cells, while we used a xenograft sarcoma tumor model. The liver uptake for different nanoparticles was reported to be $34.9 \% \mathrm{ID} / \mathrm{g}$ (nanospheres), 55.0 \%ID/g (nanodisks), $52.1 \% \mathrm{ID} / \mathrm{g}$ (nanorods), and 63.4 $\% \mathrm{ID} / \mathrm{g}$ (nanocages) at the end of 24 hours. The liver uptake of GNS is relatively low: $16.54 \pm 2.97 \% \mathrm{ID} / \mathrm{g}$ (30-nm GNS, $50 \mu \mathrm{g}), 19.77 \pm 5.76 \%$ ID/g (30-nm GNS, $200 \mu \mathrm{g})$ and $19.49 \pm 6.36 \% \mathrm{ID} / \mathrm{g}(60-\mathrm{nm}$ GNS, $200 \mu \mathrm{g})$ at the end of 48 hours. The lower liver uptake in this study might be due to dense PEGylation, which im- proves the ability of the GNS to evade phagocytosis.

Particle size also plays an important role in determining the biodistribution and clearance pathway (via liver/spleen or kidney). Nanoparticles with size less than $10 \mathrm{~nm}$ have been found to be cleared by the kidney, while the liver and spleen will quickly filter nanoparticles larger than $200 \mathrm{~nm}$.[67, 68] Consequently, a nanoparticle size between $30 \mathrm{~nm}$ to $200 \mathrm{~nm}$ has been considered to be ideal for nanoparticle accumulation in tumors through the EPR effect.[69] In this study, we compared 30-nm and 60-nm GNS with the same injection dose $(200 \mu \mathrm{g})$ and found that $30-\mathrm{nm}$ GNS have a higher tumor uptake. Future studies could be performed to investigate whether nanoparticles with a size less than $30 \mathrm{~nm}$ would have even better tumor uptake. Furthermore, our results from biodistribution of radiolabeled GNS indicate that injection dose can also affect how well the nanoparticles are taken up by the tumor. This finding is supported by the CT biodistribution data, which show that for a higher dose $(20 \mathrm{mg})$ the concentration of gold at the periphery of the tumor is almost equal to the concentration of gold within the liver and spleen (Supplementary Figure S1). This accumulation in tumors in the CT study is much higher than the accumulation observed with the lower doses used in the radiolabeled biodistribution study. One potential reason for the large differences in tumor accumulation between these experiments is RES saturation. Previous studies have shown that the RES can be saturated at high concentrations of nanoparticles, which considerably slows the clearance of those nanoparticles from the blood stream.[70] This gives them a much longer time to circulate and accumulate in tumors via the EPR effect. This finding is of importance for using nanoparticles as imaging and therapeutic agents. Further experiments could be performed to investigate the detailed relationship between nanoparticle injection dose and tumor uptake to optimize nanoparticle delivery.

The study using ${ }^{131}$ I-labeled GNS demonstrates the great potential that exists for future applications of gold nanoparticles with imaging and therapy. For example, ${ }^{124} \mathrm{I}$ is a PET radionuclide and can be attached to the gold nanoparticle surface for PET imaging, which can be used to investigate pharmacokinetics and dynamic biodistribution. In addition, ${ }^{131} \mathrm{I}$ has been used for radioisotope-based therapy.[71] The large surface area of GNS makes it a suitable carrier for radioisotope delivery including iodine-125 and astatine-211 for combined cancer imaging and treatment.[72-74] The GNS surface can also readily be modified with other radioisotopes for SPECT or gadolinium for MR imaging. This GNS nanoplatform exhibits high flexibility for combining a variety of 
imaging and therapeutic agents onto a single theranostic nanoprobe.

In this study, we developed a multifunctional theranostic GNS nanoprobe and investigated how the size, shape, and injection dose of nanoparticles affects their biodistribution and intratumoral distribution. The GNS probe can be used for in vivo biosensing and tracking using SERS, TPL, and CT at different spatial scales from whole body to the cellular level. Furthermore, we demonstrated the GNS probe's high photon-to-heat conversion efficiency and concentrated heating effect with near-infrared light, which has better performance than gold nanoshells, a widely used PTT agent. In a proof-of-principle PTT in vivo experiment, we observed effective tumor ablation after photothermal treatment. This result lays the foundation for future preclinical experiments with the multifunctional GNS nanoprobe for pretreatment plan design, intraoperative tumor margin delineation with SERS and TPL, image-guided PTT therapy, as well as therapeutic response monitoring. Such future studies are warranted to better characterize the potential of GNS as a theranostic agent for personalized cancer management.

\section{Supplementary Materials}

Figures S1-S4. http://www.thno.org/v05p0946s1.pdf

\section{Abbreviations}

GNS: gold nanostars; SERS: surface-enhanced Raman scattering; CT: x-ray computed tomography (CT); TPL: two-photon luminescence; PTT: photothermal therapy; EPR: enhanced permeability and retention; NIR: near-infrared; MPE: maximum permissible exposure; MRI: magnetic resonance imaging; PET: positron emission tomography; SPECT: single-photon emission computed tomography; pMBA: p-mercaptobenzoic acid; TEM: transmission electron microscopy; PBS: phosphate-buffered saline; FBS: fetal bovine serum; DMEM: Dulbecco's modified Eagle's medium; \%ID/g: percentage of injected dose per gram tissue; CD31: cluster of differentiation 31; PEG: polyethylene glycol; AAS: atomic absorption spectroscopy.

\section{Acknowledgments}

This work was supported by funding from Duke University Exploratory Research Fund and through the Duke Center for In Vivo Microscopy, an NIH/NIBIB National Biomedical Technology Resource Center (P41 EB015897). Yang Liu is supported by the Kathleen Zielek fellowship from the Chemistry Department of Duke University. Jeffrey Ashton is supported by an AHA Predoctoral Fellowship (14PRE20110008). Everett Moding is supported by the
National Cancer Institute of the National Institutes of Health (F30 CA177220). Jeffrey Ashton, Everett Moding, and Melodi Whitley are also supported by the Medical Scientist Training Grant (T32 GM007171). We want to thank Dr. Benjamin J. Wiley, Dr. Paolo Maccarini and Dr. Jennifer L. West at Duke University for providing access to the atomic absorption spectrometer, infrared thermal camera and gold nanoshells, respectively. The authors also would like to acknowledge the Defense Advanced Research Projects Agency (HR0011-13-2-0003). The content of the information in this paper does not necessarily reflect the position or the policy of the government, and no official endorsement should be inferred.

\section{Competing Interests}

David Kirsch is on the scientific advisory board of Lumicell Inc, which is a company commercializing intraoperative imaging. The other authors have declared that no competing financial interest exists.

\section{References}

1. Cheng Y, Morshed RA, Auffinger B, Tobias AL, Lesniak MS. Multifunctional nanoparticles for brain tumor imaging and therapy. Adv Drug Delivery Rev. 2014; 66: 42-57.

2. Wang LN, Wang YB, Li ZJ. Nanoparticle-based tumor theranostics with molecular imaging. Curr Pharm Biotechno. 2013; 14: 683-92.

3. Kim CS, Duncan B, Creran B, Rotello VM. Triggered nanoparticles as therapeutics. Nano Today. 2013; 8: 439-47.

4. Sun XL, Huang XL, Yan XF, Wang Y, Guo JX, Jacobson O, et al. Chelator-free $\mathrm{Cu}$-64-integrated gold nanomaterials for positron emission tomography imaging guided photothermal cancer therapy. ACS Nano. 2014; 8: 8438-46.

5. Zhao L, Kim TH, Ahn JC, Kim HW, Kim SY. Highly efficient "theranostics" system based on surface-modified gold nanocarriers for imaging and photodynamic therapy of cancer. J Mater Chem B. 2013; 1: 5806-17.

6. Mieszawska AJ, Mulder WJM, Fayad ZA, Cormode DP. Multifunctional gold nanoparticles for diagnosis and therapy of disease. Mol Pharmaceut. 2013; 10: 831-47.

7. Gad SC, Sharp KL, Montgomery C, Payne JD, Goodrich GP. Evaluation of the toxicity of intravenous delivery of Auroshell particles (Gold-silica nanoshells). Int J Toxicol. 2012; 31: 584-94.

8. Weissleder R. A clearer vision for in vivo imaging. Nat Biotechnol. 2001; 19: 316-7.

9. Pekkanen AM, DeWitt MR, Rylander MN. Nanoparticle enhanced optical imaging and phototherapy of cancer. J Biomed Nanotechnol. 2014; 10: 1677-712.

10. Bayazitoglu Y, Kheradmand S, Tullius TK. An overview of nanoparticle assisted laser therapy. Int J Heat Mass Tran. 2013; 67: 469-86.

11. Schlucker S. Surface-enhanced Raman spectroscopy: Concepts and chemical applications. Angew Chem Int Ed Engl. 2014; 53: 4756-95.

12. Yuan HK, Liu Y, Fales AM, Li YL, Liu J, Vo-Dinh T. Quantitative surface-enhanced resonant Raman scattering multiplexing of biocompatible gold nanostars for in vitro and ex vivo detection. Anal Chem. 2013; 85: 208-12.

13. Yuan $\mathrm{H}$, Wilson CM, Xia J, Doyle SL, Li S, Fales AM, et al. Plasmonics-enhanced and optically modulated delivery of gold nanostars into brain tumor. Nanoscale. 2014; 6: 4078-82.

14. Yuan H, Register JK, Wang HN, Fales AM, Liu Y, Vo-Dinh T. Plasmonic nanoprobes for intracellular sensing and imaging. Anal Bioanal Chem. 2013; 405: 6165-80.

15. Vo-Dinh T, Fales AM, Griffin GD, Khoury CG, Liu Y, Ngo H, et al. Plasmonic nanoprobes: From chemical sensing to medical diagnostics and therapy. Nanoscale. 2013; 5: 10127-40.

16. Liu Y, Yuan H, Vo-Dinh T. Spectroscopic and vibrational analysis of the methoxypsoralen system: A comparative experimental and theoretical study. J Mol Struct. 2013; 1035: 13-8.

17. Liu $\mathrm{Y}$, Sun $\mathrm{H}$. Electronic ground states and vibrational frequency shifts of diatomic ligands in heme adducts. J Comput Chem. 2011; 32: 1279-85.

18. Vo-Dinh T. Surface-enhanced Raman spectroscopy using metallic nanostructures. TrAC, Trends Anal Chem. 1998; 17: 557-82.

19. Zhao J, Liu Y, Fales AM, Register JK, Yuan H, Vo-Dinh T. Direct analysis of traditional Chinese medicines using surface-enhanced Raman scattering (SERS). Drug Test Anal. 2014; 6: 1063-8. 
20. Nie SM, Emery SR. Probing single molecules and single nanoparticles by surface-enhanced Raman scattering. Science. 1997; 275: 1102-6.

21. Kumar D, Saini N, Jain N, Sareen R, Pandit V. Gold nanoparticles: an era in bionanotechnology. Expert Opin Drug Deliv. 2013; 10: 397-409.

22. Xie J, Lee S, Chen XY. Nanoparticle-based theranostic agents. Adv Drug Delivery Rev. 2010; 62: 1064-79.

23. Cai QY, Kim SH, Choi KS, Kim SY, Byun SJ, Kim KW, et al. Colloidal gold nanoparticles as a blood-pool contrast agent for X-ray computed tomography in mice. Invest Radiol. 2007; 42: 797-806.

24. Liu Y, Yuan H, Kersey F, Register J, Parrott M, Vo-Dinh T. Plasmonic gold nanostars for multi-modality sensing and diagnostics. Sensors. 2015; 15: 3706-20.

25. Scaffidi JP, Gregas MK, Seewaldt V, Vo-Dinh T. SERS-based plasmonic nanobiosensing in single living cells. Anal Bioanal Chem. 2009; 393: 1135-41.

26. Alak AM, Vo-Dinh T. Silver-coated fumed silica as a substrate material for surface-enhanced Raman scattering. Anal Chem. 1989; 61: 656-60.

27. Vo-Dinh T, Meier M, Wokaun A. Surface-enhanced Raman-spectrometry with silver particles on stochastic-post substrates. Anal Chim Acta. 1986; 181: 139-48.

28. Vo-Dinh T, Hiromoto MYK, Begun GM, Moody RL. Surface-enhanced Raman spectrometry for trace organic analysis. Anal Chem. 1984; 56: 1667-70.

29. Yuan H, Khoury CG, Hwang H, Wilson CM, Grant GA, Tuan V-D. Gold nanostars: surfactant-free synthesis, 3D modelling, and two-photon photoluminescence imaging. Nanotechnology. 2012; 23: 075102.

30. Yuan H, Khoury CG, Wilson CM, Grant GA, Bennett AJ, Vo-Dinh T. In vivo particle tracking and photothermal ablation using plasmon-resonant gold nanostars. Nanomedicine: NBM. 2012; 8: 1355-63.

31. Yuan HK, Fales AM, Khoury CG, Liu J, Vo-Dinh T. Spectral characterization and intracellular detection of surface-enhanced Raman scattering (SERS)-encoded plasmonic gold nanostars. J Raman Spectrosc. 2013; 44: 234-9.

32. Liu Y, Chang Z, Yuan H, Fales AM, Vo-Dinh T. Quintuple-modality (SERS-MRI-CT-TPL-PTT) plasmonic nanoprobe for theranostics. Nanoscale. 2013; 5: 12126-31.

33. Mito JK, Min HD, Ma Y, Carter JE, Brigman BE, Dodd L, et al. Oncogene-dependent control of miRNA biogenesis and metastatic progression in a model of undifferentiated pleomorphic sarcoma. J Pathol. 2013; 229: 132-40.

34. Kirsch DG, Dinulescu DM, Miller JB, Grimm J, Santiago PM, Young NP, et al. A spatially and temporally restricted mouse model of soft tissue sarcoma. Nat Med. 2007; 13: 992-7.

35. Jackson EL, Willis N, Mercer K, Bronson RT, Crowley D, Montoya R, et al. Analysis of lung tumor initiation and progression using conditional expression of oncogenic K-ras. Genes Dev. 2001; 15: 3243-8.

36. Jonkers J, Meuwissen R, van der Gulden $H$, Peterse $H$, van der Valk M, Berns A. Synergistic tumor suppressor activity of BRCA2 and p53 in a conditional mouse model for breast cancer. Nat Genet. 2001; 29: 418-25.

37. Badea CT, Johnston S, Johnson B, Lin M, Hedlund LW, Johnson GA. A dual micro-CT system for small animal imaging. Proc SPIE. 2008; 6913: 691342.

38. Clark D, Ghaghada K, Moding E, Kirsch D, Badea C. In vivo characterization of tumor vasculature using iodine and gold nanoparticles and dual energy micro-CT. Phys Med Biol. 2013; 58: 1683-704.

39. Ashton JR, Clark DP, Moding EJ, Ghaghada K, Kirsch DG, West JL, et al. Dual-energy micro-CT functional imaging of primary lung cancer in mice using gold and iodine nanoparticle contrast agents: a validation study. PLoS One. 2014; 9: e88129.

40. Ayala-Orozco C, Urban C, Knight MW, Urban AS, Neumann O, Bishnoi SW, et al. Au nanomatryoshkas as efficient near-infrared photothermal transducers for cancer treatment: benchmarking against nanoshells. ACS Nano. 2014; 8: 6372-81.

41. Roper DK, Ahn W, Hoepfner M. Microscale heat transfer transduced by surface plasmon resonant gold nanoparticles. J Phys Chem C. 2007; 111: 3636-41.

42. Kim YH, Jeon J, Hong SH, Rhim WK, Lee YS, Youn H, et al. Tumor targeting and imaging using cyclic RGD-PEGylated gold nanoparticle probes with directly conjugated iodine-125. Small. 2011; 7: 2052-60.

43. Black KCL, Wang YC, Luehmann HP, Cai X, Xing WX, Pang B, et al. Radioactive Au-198-doped nanostructures with different shapes for in vivo analyses of their biodistribution, tumor uptake, and intratumoral distribution. ACS Nano. 2014; 8: 4385-94.

44. Singh M, Lima A, Molina R, Hamilton P, Clermont AC, Devasthali V, et al. Assessing therapeutic responses in Kras mutant cancers using genetically engineered mouse models. Nat Biotechnol. 2010; 28: 585-93.

45. Olive KP, Jacobetz MA, Davidson CJ, Gopinathan A, McIntyre D, Honess D, et al. Inhibition of Hedgehog signaling enhances delivery of chemotherapy in a mouse model of pancreatic cancer. Science. 2009; 324: 1457-61.

46. Chen $\mathrm{Z}$, Cheng $\mathrm{K}$, Walton $\mathrm{Z}$, Wang $\mathrm{Y}$, Ebi $\mathrm{H}$, Shimamura $\mathrm{T}$, et al. A murine lung cancer co-clinical trial identifies genetic modifiers of therapeutic response. Nature. 2012; 483: 613-7.

47. Liu $\mathrm{Y}$, Yuan $\mathrm{H}$, Fales AM, Vo-Dinh T. $\mathrm{pH}$-sensing nanostar probe using surface-enhanced Raman scattering (SERS): Theoretical and experimental studies. J Raman Spectrosc. 2013; 44: 980-6.

48. Liu $\mathrm{Y}$, Yuan HK, Fales AM, Vo-Dinh T. Plasmonic gold nanostar for biomedical sensing. Proc SPIE. 2014; 8957: 5.
49. O'Neal DP, Hirsch LR, Halas NJ, Payne JD, West JL. Photo-thermal tumor ablation in mice using near infrared-absorbing nanoparticles. Cancer Lett. 2004; 209: 171-6.

50. Hirsch LR, Stafford RJ, Bankson JA, Sershen SR, Rivera B, Price RE, et al. Nanoshell-mediated near-infrared thermal therapy of tumors under magnetic resonance guidance. Proc Natl Acad Sci U S A. 2003; 100: 13549-54.

51. Ko AH, Dollinger M, Rosenbaum EH. Everyone's guide to cancer therapy: How cancer is diagnosed, treated, and managed day to day. Kansas City, USA: Andrews McMeel Publishing; 2008.

52. Meier M, Wokaun A, Vo-Dinh T. Silver particles on stochastic quartz substrates providing tenfold increase in Raman enhancement. J Phys Chem. 1985; 89: 1843-6.

53. Enlow PD, Buncick M, Warmack RJ, Vo-Dinh T. Detection of nitro polynuclear aromatic-compounds by surface-enhanced Raman-spectrometry. Anal Chem. 1986; 58: 1119-23.

54. Bello JM, Stokes DL, Vo-Dinh T. Titanium-dioxide based substrate for optical monitors in surface-enhanced Raman-scattering analysis. Anal Chem. 1989; 61: 1779-83.

55. Alak AM, Vo-Dinh T. Surface-enhanced Raman-spectrometry of organophosphorus chemical-agents. Anal Chem. 1987; 59: 2149-53.

56. Karabeber H, Huang R, Iacono P, Samii JM, Pitter K, Holland EC, et al. Guiding brain tumor resection using surface-enhanced Raman scattering nanoparticles and a hand-held Raman scanner. ACS Nano. 2014.

57. Cole JR, Mirin NA, Knight MW, Goodrich GP, Halas NJ. Photothermal efficiencies of nanoshells and nanorods for clinical therapeutic applications. J Phys Chem C. 2009; 113: 12090-4.

58. Ngwa W, Kumar R, Sridhar S, Korideck H, Zygmanski P, Cormack RA, et al. Targeted radiotherapy with gold nanoparticles: current status and future perspectives. Nanomedicine. 2014; 9: 1063-82.

59. Gao N, Chen Y, Li L, Guan Z, Zhao T, Zhou N, et al. Shape-dependent two-photon photoluminescence of single gold nanoparticles. J Phys Chem C. 2014; 118: 13904-11.

60. Bentzen SM, Gregoire V. Molecular imaging-based dose painting: a novel paradigm for radiation therapy prescription. Semin Radiat Oncol. 2011; 21: 101-10.

61. Sykes EA, Chen J, Zheng G, Chan WCW. Investigating the impact of nanoparticle size on active and passive tumor targeting efficiency. ACS Nano. 2014; 8: 5696-706.

62. Doane T, Burda C. Nanoparticle mediated non-covalent drug delivery. Adv Drug Delivery Rev. 2013; 65: 607-21.

63. Chen $\mathrm{CC}, \mathrm{Wu} \mathrm{CJ}$, Yeh MK. Gold nanoparticle-based platforms as cancer-targeted molecules delivery systems. Int J Nanotechnol. 2013; 10: 840-9.

64. Beech JR, Shin SJ, Smith JA, Kelly KA. Mechanisms for targeted delivery of nanoparticles in cancer. Curr Pharm Design. 2013; 19: 6560-74.

65. Toy R, Peiris PM, Ghaghada KB, Karathanasis E. Shaping cancer nanomedicine: the effect of particle shape on the in vivo journey of nanoparticles. Nanomedicine. 2014; 9: 121-34.

66. Black KCL, Wang Y, Luehmann HP, Cai X, Xing W, Pang B, et al. Radioactive 198Au-doped nanostructures with different shapes for in vivo analyses of their biodistribution, tumor uptake, and intratumoral distribution. ACS Nano. 2014; 8: 4385-94.

67. Perez-Campana C, Gomez-Vallejo V, Puigivila M, Martin A, Calvo-Fernandez T, Moya SE, et al. Biodistribution of different sized nanoparticles assessed by positron emission tomography: A general strategy for direct activation of metal oxide particles. ACS Nano. 2013; 7: 3498-505.

68. Ding YP, Li SP, Nie GJ. Nanotechnological strategies for therapeutic targeting of tumor vasculature. Nanomedicine. 2013; 8: 1209-22.

69. Jain RK, Stylianopoulos T. Delivering nanomedicine to solid tumors. Nat Rev Clin Oncol. 2010; 7: 653-64.

70. Li D, Johanson G, Emond C, Carlander U, Philbert M, Jolliet O. Physiologically based pharmacokinetic modeling of polyethylene glycol-coated polyacrylamide nanoparticles in rats. Nanotoxicology. 2014; 8 Suppl 1: 128-37.

71. Zhang L, Chen H, Wang L, Liu T, Yeh J, Lu G, et al. Delivery of therapeutic radioisotopes using nanoparticle platforms: potential benefit in systemic radiation therapy. Nanotechnology, science and applications. 2010; 3: 159-70.

72. McLaughlin MF, Woodward J, Boll RA, Wall JS, Rondinone AJ, Kennel SJ, et al. Gold coated lanthanide phosphate nanoparticles for targeted alpha generator radiotherapy. Plos One. 2013; 8: e54531.

73. Agarwal A, Shao X, Rajian JR, Zhang HA, Chamberland DL, Kotov NA, et al. Dual-mode imaging with radiolabeled gold nanorods. J Biomed Opt. 2011; 16: 051307.

74. Xing Y, Zhao JH, Conti PS, Chen K. Radiolabeled nanoparticles for multimodality tumor imaging. Theranostics. 2014; 4: 290-306. 